\title{
Differentiated strain-control of localized magnetic modes in antidot arrays
}

\author{
Nabil Challab ${ }^{1},{ }^{*, \dagger}$ Damien Faurie ${ }^{1}{ }^{\dagger}$ Mohamed Haboussi $^{1},{ }^{\dagger}$ Adekunle O. \\ Adeyeye $^{2,3}{ }^{\dagger}{ }^{\dagger}$ and Fatih Zighem ${ }^{1 \dagger}$ \\ $\dagger^{1}$ LSPM-CNRS UPR3407, Université Sorbonne Paris Nord, 93430 Villetaneuse, France \\ $\ddagger^{2}$ Information Storage Materials Laboratory Department of Electrical and Computer \\ Engineering National University of Singapore 117576 Singapore, Singapore \\ 9 ${ }^{3}$ Department of Physics, Durham University, South Road, Durham DH1 3LE, United \\ Kingdom \\ E-mail: nabil.challab@lspm.cnrs.fr
}

\section{Nanofabrication of $\mathrm{Ni}_{60} \mathrm{Fe}_{40}$ antidot arrays}

The IL principle involves the use of interference patterns generated from two obliquely incident beam paths to expose a photoresist layer as shown in figure 1 -a $)^{1,2}$. To this end, a $127.5 \mu \mathrm{m}$ thick Kapton@ layer was first spin coated with an Ultra-i 123 I-Line positive photoresist and then mounted on one arm of a Lloyd's-mirror Interferometer (see figure 1-a)) while the second arm of the interferometer holds a mirror. Both arms of the interferometer were exposed to a $325 \mathrm{~nm}$ continuous beam (He-Cd laser). The interference between the light from the source beam and the light reflected from the mirror form a standing wave pattern with alternating maxima and minima of intensity. Subsequently, a $90^{\circ}$ rotation of the Kapton $(\mathrm{R}$ layer is performed to allow a second exposure of the photoresist. After this double exposure, the photoresist was developed in CD-26, then rinsed in DI water and dried using 
a nitrogen gun. In this condition, a dot pattern is obtained on top of the Kapton $\mathbb{R}$ layer. Then, a $20 \mathrm{~nm} \mathrm{Ni}{ }_{60} \mathrm{Fe}_{40}$ film was deposited using the RF sputtering at a fixed Ar pressure of 3 mTorr. Lift-off of the deposited film was carried out in isopropyl alcohol (IPA). Completion of the lift-off process was determined by the color contrast of the patterned $\mathrm{Ni}_{60} \mathrm{Fe}_{40}$ area. The $\mathrm{Ni}_{60} \mathrm{Fe}_{40}$ material has been chosen because of its good ferromagnetic resonance (FMR) signal and for its non-zero magnetostriction.

\section{Ferromagnetic measurements with in situ voltage-induced}

\section{strains}

Broadband ferromagnetic resonance (FMR) has been employed to detect spin waves with a zero wave vector in the antidot arrays and to study the influence of in-plane elastic strains on their energies by applying electrical voltage to the ferroelectric substrate. We remind here that this voltage induced magnetoelastic coupling is based on a two step process: first, the application of an electric field inside the ferroelectric substrate induces elastic strains to the antidot array via electromechanical effects ${ }^{3}$ and secondly, magnetostriction of the magnetic antidots induces a magnetoelastic field which can be strain (or voltage)-tuned in amplitude and direction. In these conditions, a quantitative evaluation of this magnetoelastic coupling requires both the determination of the strains induced by the ferroelectric substrate to the magnetic antidots (or to the reference continuous film) through the Kapton@ layer and the induced magnetoelastic field. This latter is directly measured via the FMR peak shifts for each detectable mode ${ }^{4}$. Concerning the strain inside the magnetic medium, it depends on whether one considers the antidot arrays or the reference continuous film. For this latter, the PZT substrate is perfectly calibrated so that macroscopic strains ( $\varepsilon_{x x}^{s u b}$ and

$\left.\varepsilon_{y y}^{s u b}\right)$ in the substrate are known and are homogeneous across the whole top surface $(15 \times 7$ $\mathrm{mm}^{2}$ ). Moreover, previous works show that the macroscopic strains are fully transmitted through the thickness of the whole system ${ }^{5,6}$. The reasons are that the continuous thin film 
is much thinner than the Kapton( R) layer (about 3 and 4 orders of magnitude) and that the interface is fully continuous. In the case of antidots, the mechanical problem is more complex. Indeed, the strain is heterogeneously distributed in the magnetic medium, because of nano-hole edges combined with the mechanical contrast between $\mathrm{Ni}_{60} \mathrm{Fe}_{40}$ and Kaptonß), as it will be illustrated in the next section. Thus, we use full-field modeling to estimate quantitatively the strain distribution.

\section{Numerical methods for magneto-mechanical behavior es- timation}

The micromagnetic simulations have been performed with the aim to locate the different magnetic modes and to calculate their frequency dependencies as a function of the applied magnetic field ${ }^{7 ?-9}$. These latter dependencies can be directly compared to the FMR experiments. To this end, a square array of 16 antidots (diameter $250 \mathrm{~nm}$ with a periodicity of $600 \mathrm{~nm}$ ) in a square cell with $2400 \mathrm{~nm}$ sides was created. Cuboid cells of volume $5 \times 5 \times 5$ $\mathrm{nm}^{3}$ have been used. The parameters used in the simulations have been obtained from the FMR analysis of the continuous film (not shown here): $M_{s}=950 \mathrm{emu}^{\mathrm{cm}}{ }^{-3}, \frac{\gamma}{2 \pi}=0.0028$ GHz.Oe ${ }^{-1}$ and the exchange stiffness $A=1.3 \times 10^{-6}$ erg.cm ${ }^{-1}$. At a first step, the LandauLifshitz-Gilbert equation was solved with a damping constant fixed at $\alpha=0.5$ for each system (CSA and SA) by applying a static magnetic field ranging from 100 to 2000 Oe by step of 50 Oe along $x$-axis allowing recording the equilibrium state. In a second step, the dynamic response of both systems is obtained by exciting the system with a short magnetic field pulse applied orthogonally which disturbs the equilibrium state. The system can thus relax following the LLG equation with a damping constant fixed at $\alpha=0.008$. The magnetic pulse has the following form $\widetilde{h}=h_{0} \operatorname{sinc}\left(2 \pi f_{\text {cut }}\left(t-t_{0}\right)\right)$, where $f_{\text {cut }}$ was fixed at $25 \mathrm{GHz}$ with a sampling frequency $f_{s}=200 \mathrm{GHz}$ giving a Nyquist frequency $f_{n}=100 \mathrm{GHz}$. Each dynamic simulation is calculated over $20 \mathrm{~ns}$ divided in 4000 stages and each stage is got by 
mean of the Runge Kutta algorithm. The Fourier transform method was used to get the resonance frequencies related to the different magnetic modes and their spatial localization. For this purpose, the magnetization configuration is stored for each elementary volume and for each stage as well as the mean value of each component. Then, the Fourier transform of the magnetization component $m_{y}=\frac{M_{y}}{M_{s}}$ is calculated to obtain the resonance frequencies. The spacial absorption at the corresponding frequencies are then calculated using the averaged value of each elementary mesh cell over the thickness, since we consider the thickness small enough to get uniform distribution of the magnetization over it.

The strain field generated in the antidots induced by the PZT substrate is determined by finite element method within COMSOL Multiphysics@. The geometry of the systems consists of a Kapton R layer of dimensions $7.5 \times 7.5 \times 5 \mu \mathrm{m}^{3}$ and a $\mathrm{Ni}_{60} \mathrm{Fe}_{40}$ magnetic antidot array of dimensions $7.5 \times 7.5 \times 0.02 \mu \mathrm{m}^{3}$ containing $8 \times 8$ nanodots periodically distributed according to both SA and CSA systems. The interface between the polymer layer and the antidots is assumed to be perfect (perfect adhesion implying continuity of the displacement and the traction vector at the interface). It is worth noting that the represented thickness of the Kapton( $\mathrm{B}$ layer $(5 \mu \mathrm{m})$ is much lower than the real thickness $(127.5 \mu \mathrm{m})$. This thickness is however sufficient to avoid possible perturbations that may be caused by the stiffer magnetic antidots on the average strain of the compliant layer. It is also worth noting that the PZT ferroelectric behavior is not modeled here, only the strain applied by the PZT to the Kapton $\mathrm{R}$ is modeled. All these justified modeling choices are motivated by optimization/reduction of the computing time. Thus, it is necessary during numerical simulations to replicate the homogeneous in-plane strains transmitted by the PZT substrate to the Kapton $\mathrm{R}$ layer. For this purpose, the following tensile-compression displacement loading will be applied to the Kapton $\mathbb{R}$ layer:

$$
U_{x}=\epsilon_{x x}^{s u b} x \quad / \quad x= \pm \frac{L}{2}
$$




$$
U_{y}=\varepsilon_{y y}^{s u b} y \quad / \quad y= \pm \frac{L}{2}
$$

$U_{x}$ and $U_{y}$ represent the displacement magnitudes applied quasi-statically to the Kapton $\mathbb{R}$ layer and $L$ is the length of the Kapton $\mathbb{R}$ layer. Regarding the spatial discretization of the calculation domain in accordance with the finite element method, the COMSOL Multiphysics automatic mesher was used. Special attention was paid to densify the mesh wherever necessary, i.e. in the neighborhoods of interfaces and free surfaces. An evolutive spatial discretization was thus carried out, utilizing a 1-degree tetrahedral Lagrangian finite element, with a minimum characteristic length of $10 \mathrm{~nm}$. This length enables to have two rows of finite elements along the thickness of the deposit, which is sufficient to accurately calculate the mean strain in the antidots. Moreover, it has been checked that the adopted meshing density enables to reach the convergence of the numerical results. In view of the relatively small deformations involved here, the calculations are carried out within the linear framework of small perturbation assumptions. Within the small ranges of strain, the materials studied behave linearly in addition to being isotropic. Once the calculations are performed, a gage zone of dimensions $3 \times 3 \mu \mathrm{m}^{2}$ was considered inside the antidot so as to avoid the disturbance induced by the free and loaded edges during the post-processing of the numerical results.

\section{Acknowledgement}

This work was supported by the French-Singaporean International Research Program (IRP CNRS-NUS Stretch-Smart) and in the frame of the ElecAcouSpin ANR project, grant ANR19-CE24-0009 of the French Agence Nationale de la Recherche (ANR). In addition, ANR and CGI (Commissariat à l'Investissement d'Avenir) are gratefully acknowledged for their financial support through Labex SEAM (Science and Engineering for Advanced Materials and devices), ANR-10-LABX-0096 and ANR-18-IDEX-000. The authors want to thank Nicolas Greneche, responsible of the MAGI server in USPN for his support during the simulations. 


\section{References}

(1) Zighem, F.; Faurie, D.; Belmeguenai, M.; Garcia-Sanchez, A.; Lupo, P.; Adeyeye, A. O. Large area periodic ferromagnetic nanowires deposited onto a polymer substrate, Appl. Phys. Lett. 2017, 111 (5), 052408-052413. DOI: $10.1063 / 1.4995397$

(2) Lu , C.; Lipson, R. H. Interference lithography: a powerful tool for fabricating periodic structures. Laser Photonics Rev. 2010, 4 (4), 568-580. DOI: 10.1002/lpor.200810061

(3) Sadat, T.; Faurie, D.; Godard, P.; Thiaudière, D.; Renault, P.-O.; Zighem, F. $90^{\circ}$ ferroelectric domain switching effect on interfacial strain mediated magnetoelectric coupling. J. Phys. D: Appl. Phys. 2020, 53 (14), 145001-145011. DOI:10.1088/1361-6463/ab6431

(4) Zighem, F.; Faurie, D.; Mercone, S.; Belmeguenai; M.; Haddadi, H. Voltageinduced strain control of the magnetic anisotropy in a $\mathrm{Ni}$ thin film on flexible substrate. J. Appl. Phys. 2013, 114 (7) , 073902-073908. DOI: $10.1063 / 1.4817645$

(5) Zighem, F.; Belmeguenai, M.; Faurie, D.; Haddadi, H.; Moulin, J. Combining ferromagnetic resonator and digital image correlation to study the strain induced resonance tunability in magnetoelectric heterostructures. Rev. Sci. Inst. 2014, 85 (10), 103905-103911. DOI: 10.1063/1.4897237

(6) Zighem, F.; El Bahoui, A.; Moulin, J.; Faurie, D.; Belmeguenai, M.; Mercone , S.; Haddadi, H.; Micro-strip ferromagnetic resonance study of strain-induced anisotropy in amorphous FeCuNbSiB film on flexible substrate. J. Appl. Phys. 2014, 116 (12), 123903-123911. DOI: 10.1063/1.4896170 
(7) Ding, J.; Tripathy, D.; Adeyeye, A. O. Dynamic response of antidot nanostructures with alternating hole diameters. EPL 2012, 98 (1), 16004-16010. DOI: $10.1209 / 0295-5075 / 98 / 16004$

(8) Gubbiotti, G.; Montoncello, F.; Tacchi, S.; Madami, M.; Carlotti, G.; Giovannini, L.; Ding, J.; Adeyeye, A. O. Angle-resolved spin wave band diagrams of square antidot lattices studied by Brillouin light scattering. Appl. Phys. Lett. 2015, 106 (26), 262406-262411. DOI: 10.1063/1.4923271

(9) Neusser, S.; Botters, B.; Becherer, M.; Schmitt-Landsiedel D.; Grundler, D.; Spin-wave localization between nearest and next-nearest neighboring holes in an antidot lattice. Appl. Phys. Lett. 2008, 93 (12), 122501-122504. DOI: $10.1063 / 1.2988290$ 